\title{
ARTIGOS
}

\section{LA SOCIOLOGÍA COMO PROFESIÓN EN ARGENTINA DESDE MEDIADOS DEL SIGLO XX \\ JUAN PEDRO BLOIS}

\section{RESUMEN}

Este artículo se propone hacer una reconstrucción de los debates e iniciativas institucionales en torno a la constitución de la sociología como una "profesión" en la Argentina desde mediados del siglo pasado. Para ello hace foco en la Carrera de Sociología de la Universidad de Buenos Aires, una institución que desde su creación en 1957 ocupó una posición central en el escenario de la sociología argentina. Apoyado en una investigación empírica más amplia (que incluyó la revisión de programas de materias, planes de estudio y otros documentos, así como la realización de entrevistas a actores relevantes), este trabajo examina las diversas tomas de posición sobre la relación de la disciplina con el mundo del trabajo que se fueron dando en distintos momentos.

SOCIOLOGÍA • PROFESIÓN • TRABAJO • ARGENTINA

\section{SOCIOLOGY AS A PROFESSION IN ARGENTINA FROM THE MIDDLE OF THE $2 \mathrm{O}^{\text {TH }}$ CENTURY}

\section{ABSTRACT}

This article proposes to reconstruct the institutional debates and initiatives around the constitution of sociology as a "profession" in Argentine, from the middle of the past century. To do so, it focuses on the career of sociology of the University of Buenos Aires. This is an institution that, since its inception in 1957, has occupied a central position in the sociology field in Argentina. Based on a broader empirical study (which included reviewing syllabus, course plans and other documents, as well as conducting interviews with relevant actors), the present study examines the various positions taken at different times regarding the relation of this discipline to the world of work. 


\section{LA SOCIOLOGIE COMME PROFESSION EN ARGENTINE A PARTIR DU MILIEU DU XX SIECLE \\ RÉSUMÉ}

L'objectif de cet article est de reconstruire les débats et les initiatives institutionnelles concernant la consolidation de la sociologie comme "profession" en Argentine, a partir du milieu du siècle dernier. A ce fin, il se penche sur le Cours de Sociologie de l'Universidad de Buenos Aires, institution qui, dès sa création en 1957, a occupé une position centrale dans le paysage de la sociologie argentine. Appuyée sur une recherche plus large (quénglobe la révision des programmes de la discipline, des plans d'études et d'autres documents, ainsi que la réalisation d'entretiens avec des acteurs significatifs), cette étude examine les diverses prises de positions sur le rapport entre la discipline et le monde du travail, adoptées à différents moments.

SOCIOLOGIE・PROFESSION・TRAVAIL・ARGENTINE

\section{A SOCIOLOGIA COMO PROFISSÃO NA ARGENTINA A PARTIR DE MEADOS DO SÉCULO XX}

RESUMO

Este artigo se propõe a reconstruir os debates e iniciativas institucionais em torno da constituição da sociologia como uma "profissão" na Argentina a partir de meados do século passado. Para isto, foca a carreira de sociologia da Universidade de Buenos Aires, uma instituição que, desde sua criação em 1957, ocupou uma posição central no cenário da sociologia argentina. Baseado em uma pesquisa empírica mais ampla (que incluiu a revisão de programas de matérias, planos de estudo e outros documentos, bem como a realização de entrevistas com atores relevantes), este trabalho examina as diversas tomadas de posição sobre a relação da disciplina com o mundo do trabalho adotadas em diferentes momentos. 
N LOS Últimos años las actiVIDAdes PROFESIONALES DE LOS SOCIÓlogos EN distintos países de la región y el mundo tuvieron una marcada expansión y diversificación. Al lado de la más tradicional inserción en las universidades o centros académicos, fue ganando presencia una serie de prácticas destinadas a responder de modo más inmediato a las necesidades de clientelas y públicos no académicos. Esas prácticas, desarrolladas en el Estado, las empresas, las consultoras o las organizaciones no gubernamentales -ONGs-, han ampliado el abanico de opciones laborales disponibles para los sociólogos, al tiempo que les han exigido una serie de saberes y destrezas diferenciados. Sin dudas, esos cambios expresaban transformaciones sociales más generales vinculadas a la llamada "sociedad del conocimiento" signada por una creciente valoración del saber técnico y una ampliación de los usos sociales de la sociología y otras ciencias sociales (DUBET, 2012).

Como se podría esperar lo anterior suscitó una serie de debates entre los sociólogos sobre las transformaciones de la disciplina y su papel en la sociedad. Por su importante repercusión en distintas latitudes, cabe aquí mencionar la polémica en torno a la "sociología pública” iniciada con la intervención de Michael Burawoy (2005) en su discurso como presidente de la American Sociological Association (PERLATTO; MAIA, 2012), pero también las diversas discusiones en torno a la función del sociólogo movilizadas por prestigiosas figuras (CALHOUN; WIEVIORKA, 2013; DUBAR, 2006; LAHIRE, 2006; TURNER, 2009). Mientras algunos saludaron 
los cambios e instaron a los sociólogos a ganar posiciones en diversos espacios de trabajo, otros se mostraron más preocupados por defender la "autonomía” de su disciplina frente a las crecientes demandas, proclives según esas miradas a ahogar el "espíritu crítico” propio de la sociología. En ese contexto, los estudios sobre los "nuevos oficios" de los sociólogos, orientados a darle una mayor visibilidad a las labores de quienes ponían en juego las herramientas de la disciplina más allá de los espacios académicos, se multiplicaron (entre otros: BLOIS, 2015; BONELLI, 1993; BRAGA, 2011; DE VENANZI, 2003; LEGRAND; VRANCKEN, 2004; MACHUCA, 2008; PIRIOU, 2006; RIBEIRO, 1999). Argentina no fue una excepción y aun cuando los debates en torno a las prácticas profesionales de los sociólogos fueron relativamente pocos (BLOIS, 2016), ha habido un creciente interés por abordar aquello que los sociólogos hacen en sus diversos ámbitos de intervención (BLOIS, 2012; RUBINICH; BELTRÁN, 2010).

Ahora bien, el desarrollo de esas prácticas no constituye un proceso sin precedentes en este país. Ya desde comienzos de los años sesenta, a medida que los primeros graduados de sociología eran formados, hubo quienes se ganaban la vida trabajando en alguna dependencia estatal o empresa (PEREYRA et al., 2016). Las cuestiones suscitadas alrededor de la constitución de la sociología como una "profesión” no son pues nuevas. El proceso más reciente de diferenciación y ampliación de las inserciones laborales de los sociólogos se inscribe en una historia previa de más larga data que lo condiciona y que en buena medida le da forma. En efecto, según creemos, la historia de una disciplina no es un pasado muerto, una serie de acontecimientos que simplemente ocurrieron y que ya no tienen incidencia alguna. Lejos de ello, cada vez que un sociólogo sale a buscar trabajo, elige un tema de investigación o negocia con un cliente los términos de una consultoría, la historia de la disciplina se reactualiza y pone en juego. Las categorías y los esquemas de percepción con los que se orienta y a partir de los cuales define sus preferencias y rechazos, tanto como las circunstancias que condicionan sus acciones, son el producto de la particular constitución histórica de la disciplina en su país. Caracterizar las diversas concepciones de la sociología que en distintos contextos movilizaron los sociólogos a la hora de definir su papel en la sociedad constituye un medio indispensable para entender la situación más reciente y dar cuenta de los dilemas o tensiones que se les plantean a los graduados en su relación con el mundo del trabajo. En este marco, este artículo se propone realizar una reconstrucción sociohistórica de algunos de los debates e iniciativas institucionales en torno a la constitución de la sociología como una "profesión” en la Argentina desde mediados del siglo pasado, momento en que se crean las primeras carreras de sociología.

Sin desconocer la existencia y gravitación de otras instituciones, el análisis estará centrado en lo ocurrido en la Carrera de Sociología de la Universidad de Buenos Aires -UBA-, un espacio que más allá de 
sus vaivenes ocupó un lugar central en el escenario más amplio de la sociología local. En efecto, desde su creación en 1957 contó siempre con la matrícula de estudiantes más elevada y el plantel docente más amplio. La enorme mayoría de los sociólogos argentinos realizó sus estudios en esta institución y varias de las más célebres polémicas o discusiones de la sociología local fueron motorizadas por sus graduados o docentes, lo que acordó a este espacio una fuerte visibilidad. Por lo demás, su ubicación en la ciudad capital en un país que tendió siempre a un fuerte centralismo reforzó esa gravitación. Si obviamente esta carrera no agota el conjunto de espacios propios de la disciplina, ofrece, dado su particular gravitación e historia, una buena base desde la que reconstruir el proceso que nos interesa.

El artículo sigue un orden cronológico. En primer lugar, se examina la etapa que va de 1957 a 1966, caracterizada por el predominio de una idea de la sociología como una "profesión" y la vocación por estimular la “sociología aplicada”. A continuación, se reconstruye el período 1966-1974, signado por la proliferación de concepciones muy diversas sobre la disciplina y la creciente gravitación de quienes buscaban conectar de manera más estrecha la práctica de la sociología con la militancia política. En tercer lugar, se aborda el momento de repliegue iniciado a mediados de los años setenta con la instauración del autoritarismo estatal y la creciente preocupación de los sociólogos por su inserción laboral. Finalmente se examina la etapa iniciada con la vuelta de la democracia y el predominio, en buena medida aún hoy vigente, de una mirada que tiende a deslegitimar las prácticas profesionales no académicas precisamente en un momento de expansión y diferenciación. ${ }^{1}$

\section{LA CREACIÓN DE UNA CARRERA Y LA “PROFESIONALIZACIÓN" DE LA SOCIOLOGÍA (1957-1966)}

La creación de la primera carrera de sociología en la Universidad de Buenos Aires -UBA- a mediados del siglo pasado constituye un parteaguas en la historia de la disciplina en la Argentina. Aun cuando habían existido diversos intentos de avanzar en el proceso de institucionalización (BLANCO, 2006; GONZÁLEZ BOLLO, 1999; PEREYRA, 2005), la enseñanza de la disciplina a través de una licenciatura [bachalerado], así como la posibilidad de expedir títulos reconocidos por el Estado, conllevó un nuevo estatus para la disciplina en el seno de la universidad y más allá de sus claustros. La sociología ya no se limitaría a ser una asignatura o materia auxiliar de otras carreras, a cargo de profesores cuya ocupación principal no siempre tenía que ver con la disciplina (GIORGI, 2010).

Esa iniciativa estuvo signada por la creencia en la receptividad que el Estado y la sociedad en su conjunto podrían tener para la 
"nueva ciencia" y los futuros graduados. Los problemas laborales, educativos, sanitarios y asistenciales que las diversas agencias públicas debían abordar, tanto como la gestión de los recursos humanos y los estudios de mercado y consumo en el sector privado, aparecían, junto con el trabajo en instituciones académicas, como potenciales áreas de intervención para los nuevos profesionales (GERMANI; GRACIARENA, 1958). Semejante expectativa estaba en consonancia con lo que ocurría en otros países donde, en el marco de una renovada fe en la razón y en las ciencias sociales, la figura del "experto" en "desarrollo" y "planificación” ganaba presencia (PICÓ, 2003).

Según Gino Germani (1956/1962), el principal impulsor del nuevo espacio, en los países centrales donde la disciplina se hallaba más desarrollada se habían configurado tres "subdivisiones" o campos disciplinarios. Por un lado, estaba la "sociología teórica” que, con una orientación nomotética, estudiaba los hechos sociales para formular proposiciones de validez general. Por otro, estaba la "sociología descriptiva” que, con una orientación idiográfica, tenía por objeto conocer una sociedad particular. Finalmente, estaba la "sociología aplicada” cuyo propósito principal era preparar soluciones inmediatas para los “problemas sociales” (GERMANI, 1956/1962, p. 65). Si las primeras dos subdivisiones tenían como destinatarios principales a los propios sociólogos, la última tenía como público principal a las instituciones de la sociedad civil y, de manera protagónica, al Estado. Ahora bien, según aclaraba, esa "división del trabajo" no debía afectar la "unidad de la sociología”. En efecto, pese a sus distintas orientaciones y espacios de inserción, las diferentes subdivisiones compartían los mismos fundamentos epistemológicos y metodológicos, aquellos propios de la “ciencia en general” (GERMANI, 1956/1962, p. 63). Por ello, lo que debía haber entre ellas eran relaciones de mutua colaboración e intercambio, en el marco de la "conexión más estrecha posible”. Dar soluciones a un público o cliente no constituía una desviación u obstáculo para el ejercicio de la disciplina. Lejos de ello, esa labor podría constituir un valioso insumo para la disciplina en su conjunto. De hecho, según el sociólogo italiano, la sociología aplicada:

[...] no se halla en contraste con la posibilidad de aprovechar sus resultados o su metodología, desde el punto de vista teorético [o académico]. Al contrario, este tipo de investigación puede ofrecer oportunidades para realizar observaciones e incluso experimentos en condiciones particularmente favorables [...] lo importante es mantener una estrecha conexión con la teoría sociológica que habrá de proporcionar todo el encuadre de sus investigaciones. (GERMANI, 1956/1962, p. 65) 
Inspirados en esa visión de la disciplina, Germani y sus colaboradores buscaron promover un perfil de graduado que, además de desenvolverse con soltura en la esfera académica, fuera capaz de desarrollar sus actividades más allá del sistema científico. En ese sentido, el plan de estudios de la novel carrera contempló la oferta de un "Certificado de especialista en Sociología aplicada", destinado a aquellos graduados interesados en insertarse como "expertos en problemas sociales” (FACULTAD DE FILOSOFÍA y LETRAS, 1962). La primera orientación disponible era "Psicología social", oferta que aprovechaba las materias de la también flamante Carrera de Psicología (DAGFAL, 2009). Pero el plan dejaba abierta la posibilidad de establecer nuevas especialidades. Según su mirada, había un conjunto variado de funciones disponibles para los futuros graduados que, sin embargo, permanecían ocupadas por personas que no contaban con una formación adecuada o suficiente (GERMANI; GRACIARENA, 1958). En función de ello, las autoridades de la nueva institución buscaron sellar convenios con organismos estatales vinculados a la planificación, ofreciéndoles asesoramiento y recursos humanos para sus diversas iniciativas (GERMANI, 2004). Germani intentó asimismo incorporar docentes con experiencia fuera de la academia, como fue el caso de Jorge García Bouza, un abogado especialista en trabajo cuyas primeras intervenciones buscaban transmitir sus experiencias profesionales. En conjunto, esas acciones eran parte de una preocupación más general por estrechar la cooperación con entidades públicas y privadas con el fin de permitir “a los estudiantes realizar prácticas de aplicación dentro de aquellos organismos en que estarán destinados a actuar como profesionales después de haber concluido sus estudios" (GERMANI; GRACIARENA, 1958, p. 6). Por lo demás, a los encuentros con empresarios y publicistas que el sociólogo italiano había promovido en los años previos (MILANESIO, 2014), con vistas a convencerlos del potencial de la sociología a la hora de conocer los deseos de los consumidores, se sumaba ahora el intento por dar a conocer las actividades de la nueva institución a través de la prensa y los medios de comunicación. ${ }^{2}$

Ahora bien, varios fueron los factores que obstaculizaron la consolidación de ese proyecto. Por un lado, se dio un vertiginoso

En más de una oportunidad la revista Primera Plana se hizo eco de las actividades de la Carrera de Sociología de la UBA. En una de esas notas, titulada "Dos mi doscientos argentinos bajo la lupa de los sociólogos", presentaba algunas de las encuestas desarrolladas por German y sus colaboradores con abundantes detalles $y$ extensión (Primera Plana. 11 de diciembre de 1962). aumento del número de alumnos que rápidamente se ubicó en torno a los 500 anuales (RODRÍGUEZ BUSTAMANTE, 1979). Ese aumento, que superaba en mucho lo previsto y deseado por el sociólogo italiano (GERMANI, 2004), obligó a la contratación de un amplio grupo de jóvenes docentes con dedicaciones de tiempo parcial que no tenían demasiada experiencia en las tareas de investigación ni en el ejercicio "aplicado" de su disciplina. En esas condiciones, la enseñanza no podía adoptar el perfil más "práctico" que se había buscado y debió conformarse con clases más expositivas y librescas (GERMANI, 1970). Por el otro, los 
alumnos, inmersos en un proceso de creciente politización (RUBINICH, 1999), se mostraban cada vez más disconformes con el perfil de la Carrera. Para ellos, la sociología no era ni debía ser asumida como una “profesión” y era preciso impulsar la enseñanza de bibliografía “crítica”, que les permitiera participar de los grandes debates ideológicos que agitaban el espacio público, en detrimento de los contenidos más técnicos vinculados a la enseñanza de la metodología (BLOIS, 2015). Asimismo, la receptividad de las diversas dependencias estatales hacia la nueva carrera y sus producciones estuvo lejos de lo esperado; algo que quedó expresado de manera patente a partir del golpe de Estado de 1966y la intervención que se dio en las universidades. La Carrera sufrió entonces un duro embate, que desarticuló buena parte de las iniciativas que se venían llevando a cabo y obligó a un profundo recambio de su cuerpo de profesores. Cabe aclarar que el limitado apoyo estatal no era una particularidad de la sociología, siendo parte de la dinámica más general de un Estado que, a diferencia de lo que paralelamente ocurría en casos como el brasileño, mexicano o chileno (BLANCO; JACKSON, 2017; BEIGEL, 2010), no se destacaba por su apoyo sostenido a los emprendimientos científicos y culturales (SIGAL, 1991).

\section{LA POLITIZACIÓN Y EL EJERCICIO PROFESIONAL DE LA SOCIOLOGÍA (1966-1974)}

Con todo, en lo sucesivo la cuestión laboral no desapareció del horizonte de preocupaciones. En aquellos años, cuando los sociólogos pensaban sobre sus opciones profesionales predominaba una extendida presunción según la cual no había suficientes trabajos para ellos. Muestra de ello, según una nota de la popular revista Confirmado de septiembre de 1969, la situación no era la más propicia para quienes iniciaban la carrera de sociología con la idea de asegurarse un futuro laboral más o menos aceptable. De acuerdo a esa nota,

\footnotetext{
[...] las perspectivas que la Argentina abre a un sociólogo recién recibido no son capaces de entusiasmar a nadie [...] Los sociólogos son profesores bastante mal pagos en las universidades; a veces trabajan en tareas que nadie puede definir bien [en] organismos estatales, en los departamentos de obra social de las municipalidades [o] en las oficinas de selección de personal de algunas grandes empresas. (CARRI, 2015, p. 93)
}

Ante tal panorama y preocupados por el destino de los jóvenes graduados, había figuras que, en línea con las orientaciones que habían guiado la creación de la Carrera, llamaban a una proactiva promoción de la sociología como insumo fundamental para el desarrollo de 
diversas instituciones y a una decidida búsqueda de nuevas clientelas y empleadores.

Para estos sociólogos, era preciso evitar dos riesgos contrapuestos. De un lado, había que impedir la consolidación de la sociología como una labor puramente académica, cerrada y autorreferencial, en la que la producción de conocimiento apareciera como un puro fin en sí mismo. Del otro, era imperioso evitar una “ideologización” excesiva que desconociera la especificidad de sus saberes y métodos, confundiéndola con la política y la lucha ideológica. Ambos peligros, con todas sus diferencias, compartían el hecho de desaprovechar el potencial de la disciplina como fuente de información y análisis racional de las sociedades contemporáneas.

La asunción de la sociología como una práctica estrictamente académica, de un trabajo sin otros destinatarios que los propios pares, reforzaba, según estos sociólogos, un pernicioso "círculo vicioso": en la medida en que la sociedad no conocía lo que la sociología podía hacer por ella, no atraía hacia sí las preocupaciones de los sociólogos; en la medida en que los sociólogos eran ajenos a las preocupaciones de la sociedad, reforzaban el desconocimiento social de su disciplina y recaían en una labor autorreferencial. En esas condiciones, según Manuel Mora y Araujo, un joven graduado que había realizado una maestría en FLACSO y que se desempeñaba en la Fundación Bariloche:

\begin{abstract}
[...] por un lado la sociología gira como una bola en el vacío, no se alimenta del resto de la sociedad ni la alimenta, no contribuyendo, por lo tanto, a cambiarla; por otro lado, la sociedad ignora la sociología, no la acepta enteramente como una actividad legítima, contribuyendo a que la disciplina resulte institucionalmente inestable, carezca de recursos, sea marginal. (MORA Y ARAUJO, 1971, p. 125)
\end{abstract}

Ahora bien, según estos sociólogos, la conexión directa de la disciplina con la práctica política no era la respuesta. De hecho, esa conexión podía inducir una misma impotencia de la disciplina en su relación con la sociedad ya que, en ese caso, el sociólogo podría intervenir como "ciudadano" o "militante" pero al costo de resignar su contribución "específica” en tanto "sociólogo". En ese marco, contra los discursos más radicalizados, afirmaban que el ejercicio profesional de la disciplina no estaba en contradicción con la idea de "compromiso", idea de gran ascendiente en el escenario intelectual del momento (SIGAL, 1991). Lejos de ello, el trabajo sobre problemáticas "concretas", propio de la "sociología aplicada”, favorecía una acción "real” sobre las "urgencias” y “malestares” del presente. Los efectos de cualquier acción "práctica”, por más limitada o reformista que fuese su orientación, 
resultaban siempre más valiosos que cualquier "discurso" por más crítico o revolucionario que fuera. En ese sentido, Adolfo Critto, un profesor de la Universidad Nacional de Córdoba que se había doctorado en Columbia bajo la guía de Robert Merton y Paul Lazarsfeld (GRISENDI, 2014), señalaba

\begin{abstract}
[...] una vez, hablando de una investigación social que yo hacía en función de un programa de desarrollo comunitario, se levantó un estudiante y me dijo: 'Doctor, pero eso que usted está haciendo es perjudicial, porque eso puede aliviar mucho la situación de esa gente, y en consecuencia no se va a producir la "revolución". Yo diría que hay dos tipos de compromiso de cambio, y eso lo he sentido muy, muy violentamente, el compromiso de cambio dentro de esta sociedad, y el compromiso de cambio de esta sociedad para hacer otra [...] yo prefiero comprometerme con esta sociedad y cambiar esta sociedad, y eso es, creo, de lo que va la sociología aplicada. Es una sociología de metas inmediatas. (CRITTO en DE ÍMAZ et al., 1966, p. 111-112)
\end{abstract}

Pero los beneficios no se limitaban al ámbito “práctico”. Lejos de ello, según estos sociólogos, las preguntas y desafíos intelectuales sugeridos por las problemáticas planteadas por las clientelas podrían estimular la "imaginación científica” y la generación de conocimientos y enfoques originales. En ese sentido, Torcuato Di Tella (1967), un sociólogo formado en Inglaterra y uno de los más estrechos colaboradores de Germani, recordaba que las “revoluciones científicas”, entre las que mencionaba el psicoanálisis, el keynesianismo y el marxismo, no se habían producido en el marco de las universidades, fruto de una búsqueda "pura” de conocimiento; sino que sólo se habían dado cuando una "tradición científica" había encontrado y procurado responder a las “necesidades prácticas” de la sociedad. Es decir, cuando los científicos, abandonando su torre de marfil, se habían comprometido con la suerte de sus contemporáneos y sus problemáticas (sean las dolencias psicológicas en el caso de Freud, la profunda crisis del capitalismo en el de Keynes, o la organización y emancipación de una nueva clase social en el de Marx). Como vemos, en la búsqueda de su "paciente", de los actores e instituciones que necesitaban sus servicios (y la lista mencionada era larga: dependencias estatales, empresas, sindicatos, cooperativas, etc.), la disciplina se jugaba mucho más que la adecuada inserción de sus practicantes en el mercado laboral.

Ahora bien, la “sociología aplicada” suponía una concepción según la cual la sociología era una empresa "neutral” susceptible de servir los fines más diversos. Es decir, en sí misma, la disciplina no daba elementos para orientar el sentido o dirección de los cambios en la sociedad. Lo que 
ella podía hacer era favorecer la elección de los medios más adecuados para ciertos fines, señalar la inviabilidad de ciertas decisiones, ampliar el margen de opciones disponibles para el decisor. Contra quienes veían en la sociología una disciplina comprometida con ciertas orientaciones o preferencias ideológicas, Mora y Araujo alertaba:

\begin{abstract}
[...] existe una tendencia a creer que si la sociología puede ser útil a algo, es a la defensa del statu quo. Esta creencia, muy difundida en los sectores de "izquierda", es tan prejuiciosa como la creencia difundida en los sectores de "derecha", de que la sociología no es más que un pretexto para producir mentalidades subversivas. Creo que la sociología puede ser útil para servir objetivos pro statu quo como anti statu quo, objetivos reformistas como revolucionarios, de derecha tanto como de izquierda. La sociología puede servir para predecir la ocurrencia de guerrillas y controlarlas mejor, pero puede ser igualmente útil a las guerrillas para establecer si sus objetivos son plausibles o descabellados, si los efectos esperados ocurrirán o no. (MORA Y ARAUJO, 1971, p. 134)
\end{abstract}

Con todo, esa neutralidad no implicaba, según estos sociólogos, una sumisión inmediata a los dictados de la clientela o empleador. Lejos de ello, volcar la actividad sociológica hacia el asesoramiento de instituciones de diverso tipo no debía hacer del sociólogo un individuo sin margen de maniobra que asumía la realización de los encargos en las condiciones y ritmos fijados por quienes financiaban su trabajo. La sociología, como cualquier profesión, implicaba el despliegue de un conjunto de normas o principios morales y cognoscitivos, propios de la disciplina, que era preciso respetar: el reconocimiento de tiempos o plazos mínimos que garantizaran cierto estándares de calidad, la realización de indagaciones de mayor alcance, susceptibles de poner en juego ideas teóricas más complejas, la búsqueda de inspiración y soporte en las investigaciones desarrolladas en la academia, etc. Sólo así la "sociología aplicada" estaría en condiciones de promover el desarrollo e innovación de la disciplina en su conjunto que tantas expectativas despertaba.

Ahora bien, la creciente politización y las expectativas sobre una posible transformación radical de la sociedad que signaba los medios intelectuales y universitarios, no ofrecía el marco más receptivo para esas ideas (SIDICARO, 1993). Para dar cuenta de ese clima cabe aquí referir una nota realizada por el popular semanario Panorama, que en 1971 convocó a un conjunto de sociólogos para debatir sobre la sociología como carrera universitaria y profesional. Con el fin de introducir la discusión, los periodistas citaban los datos de una "encuesta piloto" realizada por la misma revista, según la cual una muy amplia mayoría 
de quienes decidían estudiar sociología lo hacían buscando "algún tipo de política con perspectiva revolucionaria”, anhelando un título de "revolucionario" mucho más que uno de "sociólogo". Si semejante introducción no fue objetada por los participantes, hubo quienes, cómodos con esa caracterización, buscaron dar cuenta de las razones de semejante situación. Así Ricardo Sidicaro, un joven graduado que se ganaba la vida como docente de la Carrera de la UBA, afirmaba:

\begin{abstract}
Pienso que el rol de sociólogo es lo suficientemente triste como para que nadie [...] pueda llegar a plantearse cuál es su futuro [...] Hace poco un profesor de la carrera reunió a un grupo de alumnos de ingreso y les dijo que no tenía sentido que estudiaran Sociología porque no iban a tener posibilidades ocupacionales. Entonces, alguien se paró y dijo: "en realidad, nosotros no venimos acá para ser sociólogos, sino para hacer la revolución”. Con todo lo correcta que era la respuesta a una mala proposición, yo comentaba el otro día con mis alumnos que indudablemente estos jóvenes que hoy se orientan hacia la política, tiempo atrás hubieran pecado de idealistas. Ahora serían aún más idealistas si revelaran que quieren trabajar de sociólogos. Dentro de cinco años, esta probabilidad será todavía menor, salvo que el sistema [...] encuentre formas de ubicarlos: es decir lugares donde los sociólogos puedan convencer a los villeros de que es bueno que los desalojen, o que trabajen para organismos represivos [o que se inserten para] ayudar a vender jabones [en alguna empresa de análisis de mercado]. (SIDICARO en Panorama, 1971, p. 41)
\end{abstract}

En ese marco, quienes promovían la politización de la sociología, aun cuando pudieran hacerlo desde diversas posiciones partidarias, confluyeron en la recusación de la problemática laboral, denunciando de modo recurrente el ideal de la "neutralidad valorativa". Para ellos, todo discurso, incluido el de la sociología, era un discurso irreductiblemente "ideológico" que o bien operaba a favor del mantenimiento del orden social o bien lo hacía a favor de su crítica y superación. Ninguna pretendida "objetividad científica” podía apartar al sociólogo de una toma de posición frente a las luchas que agitaban su sociedad. Así, por ejemplo, según Eliseo Verón, un sociólogo que se desempeñaba en el Instituto Torcuato Di Tella y que se había convertido en uno de los más convencidos críticos de Germani (NOÉ, 2005), la asunción de la sociología como una "profesión", no era más que una coartada que buscaba poner "la disciplina a cubierto de la política y la ideología", ocultando su compromiso con los sectores dominantes y las estructuras de poder vigentes (VERÓN, 1974, p. 22). 
Como podría preverse, la labor del sociólogo académico como "técnico" o "experto" inserto en el Estado o en el sector privado suscitaron un profundo rechazo. Es que el sociólogo, según estas miradas, debía asumir su vocación como un ejercicio crítico de todo poder explotador y comprometerse con la emancipación de los sectores dominados. Su única audiencia legítima eran quienes estaban comprometidos con una transformación radical de la sociedad. En la medida en que el Estado y las empresas privadas no estaban consustanciadas con esa meta, la sociología debía construirse contra ellos. Así, Verón, recusando la convocatoria a trascender los muros universitarios en busca de nuevas clientelas, no se ahorraba las ironías:

\footnotetext{
El sociólogo ofrece ahora sus servicios en un mercado más amplio. El conocimiento sociológico puede beneficiar a todos, permite implementar cualquier política y cualquier ideología. El sociólogo deja su tarjeta, con la esperanza de conseguir trabajo. La afirmación explícita y consciente de la sociología como una tecnología que está más allá (o más acá) de los conflictos y la lucha ideológica y que cualquiera puede comprar [vuelve al sociólogo] un tecnócrata que confiesa abiertamente que al hablar del "cambio", no piensa en un cambio determinado: hacerlo podría atemorizar a muchos clientes potenciales. (VERÓN, 1974, p. 64)
}

Si los defensores de la "sociología aplicada” habían concebido al trabajo en diversas esferas o instituciones como la manera en la que el sociólogo podía comprometerse con su sociedad, esa tentativa resultaba aquí fuertemente censurada. La "tecnología sociológica”, tal como denunciaba Roberto Carri, otro reconocido docente de la Carrera, no era otra cosa que una "tecnología de la dominación” que, en lugar de vincularse con los sectores subalternos y acompañarlos en su organización y lucha contra la opresión, se consagraba a la "elaboración de recetas técnicas” para aliviar las tensiones sociales y garantizar la pervivencia de un orden injusto (1969, p. 61). Cualquier cambio gradual o reformista, como el que reivindicaban los promotores de la "sociología aplicada”, era recusado como una postergación y obstáculo para los cambios fundamentales que la hora exigía.

En ese marco, por supuesto, la transición al mundo del trabajo no se veía facilitada ni estimulada. Tampoco resultaba favorecida

Cabe destacar que en esos años, los graduados de Psicología de la UBA se embarcaron en una acción que buscaba garantizar su derecho a ejercer la clínica psicoanalítica contra el monopolio legal de los médicos psiquiatras (DAGFAL, 2009). la posibilidad de realizar una acción corporativa análoga a aquella desarrollada por otras disciplinas ${ }^{3}$ en función de garantizarse ciertas tareas como jurisdicciones propias. Pero lo que interesa destacar aquí es el fortalecimiento de una mirada que, construida en oposición a la idea de la "sociología aplicada", asumía el trabajo para un cliente o empleador como el abandono sin atenuantes de todo compromiso con 
la mejora y la transformación social. El sociólogo que aceptaba trabajar en esas condiciones era denunciando como un "tecnócrata” al servicio de los poderosos y del status quo.

Por supuesto, no es que los sociólogos no trabajasen en diversas esferas laborales. Incluso algunos de los más entusiastas defensores de la politización de la sociología debían ganarse la vida y para ello se empleaban en alguna dependencia estatal o empresa; pero esas inserciones no eran jerarquizadas como espacios legítimos de actuación, siendo a veces ocultadas o puestas en un segundo plano. En la Carrera de la UBA no faltaban las materias optativas (vinculadas a temáticas de planeamiento, salud, educación, etc.) que proyectaban al sociólogo como un "cuadro técnico" para el Estado pero, de acuerdo a los discursos críticos del momento (que esas materias muchas veces compartían), su cabal inserción dependía, o estaba a la espera, de la llegada de un gobierno comprometido con la promoción de cambios de tipo revolucionario (algo que, según se probó, no siempre estaba al alcance de la mano...).

\section{EL REPLIEGUE DE LA SOCIOLOGÍA Y LA PREOCUPACIÓN POR LA INSERCIÓN LABORAL (1974-1983)}

La instauración de la persecución y el autoritarismo político a partir de 1974, con el giro del gobierno peronista primero y luego con la instalación de la dictadura militar en 1976, clausuró buena parte de los acalorados debates en torno a lo que la sociología era y debía ser. Mientras un buen número de sociólogos debió exiliarse, las instituciones propias de la sociología sufrieron un duro revés. La Carrera de la UBA fue clausurada y luego reabierta en condiciones irreconocibles, con un conjunto de docentes de escasos antecedentes y una matrícula muy reducida. ${ }^{4}$ Su separación de la Facultad de Filosofía y Letras y su emplazamiento en el sótano de la Facultad de Derecho no ocultaba el desprecio que las nuevas autoridades tenían por una carrera que pudo haber sido cerrada (BLOIS, 2009; PEREL et al., 2006; RAUS, 2007). Al mismo tiempo, una buena parte de las carreras que habían sido creadas en la zona metropolitana y en otras regiones del país discontinuaban sus labores (LIEDKE FILHO, 1991). Los centros privados de investigación, ámbitos surgidos en los años previos como respuesta a las intervenciones gubernamentales en las universidades, pudieron continuar sus labores gracias al apoyo de fundaciones extranjeras pero sólo pudieron hacerlo en el marco de un bajo perfil que no atrajera la atención de las autoridades estatales. La reserva era tal que ese conjunto de instituciones aparecía para quienes lo frecuentaban como una "universidad de las catacumbas” (BRUNNER; BARRIOS, 1987; HEREDIA, 2011). ${ }^{5}$

Sin embargo, la preocupación por las posibilidades laborales de los sociólogos no perdió vigencia. Difícilmente podría ser de otro modo

4

Mientras en 1972 había casi 2800 alumnos, hacia 1980 esta cifra apenas superaba los 500 (BLOIS, 2009).

5

Los centros más activos eran el Instituto de Desarrollo Económico y Social-IDES-, el Centro de Estudios Urbanos y Regionales-CEUR-, el Centro de Estudios de Población -CENEP-, el Centro de Investigaciones sobre el Estado y la Administración -CISEA-, el Centro de Estudios de Estado y SociedadCEDES- y el Centro de Investigaciones en Ciencias Sociales -CISCO. Para una comparación de la situación de estas instituciones en Brasil y Argentina, puede verse Blois (2015). 
si se recuerda que hasta 1978, más de 2200 individuos habían terminado sus estudios en sociología en la ciudad de Buenos Aires (BIALAKOWSKY et al., 1982). Si ya en el período anterior había un extendido consenso sobre las dificultades de inserción de los graduados, la nueva etapa, signada por la desarticulación y deterioro de las instituciones disciplinarias, así como por las sospechas de las autoridades sobre el carácter "subversivo" de la sociología, no mejoraba el panorama.

En ese marco, fruto de los esfuerzos de un conjunto de sociólogos preocupados por el desarrollo profesional de la disciplina, en 1975 se produjo la creación del Colegio de Graduados de Sociología -CGS-, una institución que buscaba servir como espacio de reunión y defensa de los intereses "corporativos" de sus integrantes. La organización de los sociólogos podría facilitar, según el diagnóstico de sus impulsores, la jerarquización de sus saberes y su inserción profesional, ofreciendo para ello un espacio de discusión e intercambio, donde la circulación de informaciones sobre oportunidades laborales sería frecuente. Quizá de modo imprevisto el contexto represivo acabó fortaleciendo el accionar del CGS, constituido en uno de los principales refugios para quienes habían sido excluidos de los espacios oficiales.

El CGS desarrolló una amplia oferta de cursos de "actualización profesional”. Dentro de la oferta fueron sin duda aquellos de perfil más “técnico", centrados en la metodología y técnicas de investigación, los que mayor interés concitaron entre un público que buscaba adquirir una formación capaz de mejorar sus posibilidades laborales, sea en el mundo privado, sea en la burocracia estatal. Para varios, que habían estudiado en la UBA en su período de máxima politización y que en consonancia con ello habían desdeñado las materias metodológicas, la realización de esos cursos constituía una oportunidad para compensar lo que ahora veían como una "falta” o déficit en su formación. Lejos de las miradas que en los años anteriores habían cuestionado la búsqueda de nuevas clientelas, el CGS defendía la constitución de la sociología como una "profesión" como cualquier otra: la "ideologización" que había sacudido a la disciplina en el pasado inmediato aparecía ahora como un "desajuste” que podía llevar "al extrañamiento (alienación) del rol específico" del sociólogo y a su "frustración" (BIALAKOWSKY et al., 1982, p. 4). Según esa mirada, el desempeño profesional no sólo debía constituir "una forma elemental de proveer a nuestro sustento", sino que debía orientarse a reforzar el "autoestima” de los sociólogos y a "servir a la comunidad en la medida de nuestras posibilidades” (BIALAKOWSKY et al., 1982, p. 1).

El nuevo contexto y el deterioro de las instituciones académicas promovió la recuperación de las ideas de aquellas figuras que, en el pasado, habían defendido la inserción de los sociólogos en el Estado y las empresas. Con todo, esa recuperación no debería ocultar las 
diferencias entre la posición alentada por el CGS y aquéllas propias de los años previos. Antes el análisis de lo que los sociólogos podían hacer para ganarse la vida había estado en función de una pregunta más general sobre el papel o función de la disciplina en la sociedad. Y, como vimos, las respuestas no habían sido modestas: quienes promovían la “sociología aplicada” confiaban en la constitución de la sociología como una fuerza intelectual (para algunos comparable con el psicoanálisis, el keynesianismo o el marxismo) capaz de contribuir en la racionalización y mejora de la acción de la sociedad sobre sí misma. En su visión, el desarrollo de la sociología era una de las condiciones necesarias para el desarrollo de la sociedad. Tampoco habían sido modestos quienes, en contra de esas miradas, preferían ver en la disciplina un conjunto de insumos capaces de alentar la crítica al orden social vigente y su superación en el marco de una serie de transformaciones revolucionarias. Ahora bien, más allá de sus diferencias, esas posiciones compartían el hecho de reconocer en la sociología una misión trascendente que excedía por mucho la pura preocupación por la problemática laboral. En la nueva etapa, sin las certezas que habían sustentado aquellas visiones -la creencia en el desarrollo y modernización de las sociedades, por un lado, la confianza en la capacidad redentora de los pueblos y la clase obrera, por el otro-, la pregunta de los sociólogos por su disciplina se hacía más humilde y acotada: ya no se trataba de saber lo que la disciplina podía hacer por la sociedad sino que lo que ahora importaba saber era de qué podían trabajar los sociólogos. Se producía así un desdibujamiento de las ideas sobre la utilidad o función de la disciplina, así como un debilitamiento de la creencia en la posibilidad de comprometerse con fines más ambiciosos o amplios: ya no se trataba de cambiar u orientar la sociedad a partir de la sociología sino de adaptar la sociología a las necesidades de la sociedad.

Expresión de ello, en 1980 el CGS encargó la realización de una encuesta sobre el "espectro ocupacional del licenciado en sociología”, una iniciativa que buscaba echar luz sobre la realidad laboral de los graduados. Según sus impulsores, un conocimiento más ajustado de esa realidad podría señalar los "requisitos que una currícula educativa debiera contener" en función de las "probables inserciones laborales y de una más clara y explícita visualización de las posibilidades de ejercicio profesional” (BIALAKOWSKY et al., 1982, p. 12). Como vemos, la reflexión en torno a las prácticas laborales de los sociólogos asumía, en ese marco, la forma más habitual de los estudios sobre graduados universitarios, orientada a medir la pertinencia de la formación brindada por las carreras y su eventual ajuste o desajuste en relación con las demandas del mercado laboral.

El giro era también palpable en alguna de las figuras que en el pasado habían impulsado el desarrollo de la "sociología aplicada”. Así, 
en 1980, Di Tella volvía a insistir en su llamado a trascender la inserción académica y a emplearse en diversas instituciones, pero lo hacía con un tono más pragmático centrado en la preocupación por las posibilidades laborales de los graduados. Si su blanco principal era la experiencia de radicalización de los años sesenta que había conspirado contra el desarrollo de la sociología como una "profesión”, su prédica, que dejaba de lado los modelos ofrecidos por el psicoanálisis, el keynesianismo o el marxismo, ya no se ilusionaba con el lugar que la sociología, gracias a la participación de los sociólogos en diversos ámbitos, podría ocupar en la sociedad. La "sociología aplicada” no prometía ahora "revoluciones científicas", siendo ante todo defendida como un medio para "ganarse la vida” en un contexto particularmente difícil para la disciplina.

\footnotetext{
Hay una tendencia en los que se aproximan a la sociología a desvalorizar estas técnicas, sea porque son menos estimulantes a la imaginación que las interpretaciones generales de la sociedad o de la política, o porque se considera que sirven sólo para poner remiendos a la sociedad, o para hacer ganar dinero a quienes contratan estos trabajos. Aunque esto pueda ser cierto, no lo es menos que de alguna manera hay que ganarse la vida, y estas especialidades son de las más prometedoras en este sentido. En la medida que el sociólogo deja de lado su obsesión con la utopía, puede aceptar más las limitaciones de la condición humana -que incluye la propia- y aceptar ser, en una parte muy apreciable de su actividad, una rueda en el engranaje de la sociedad. El sociólogo, como cualquier otro profesional, debe ser capaz de asesorar y "curar" al paciente o cliente, sin preguntarse necesariamente si ese cliente va a misa todos los días, o si tiene convicciones políticas que lo colocan en la vanguardia del cambio social. (DI TELLA, 1980, p. 312)
}

\section{LA CONSTRUCCIÓN DE UNA CARRERA DE ESPALDAS AL MUNDO DEL TRABAJO DESDE LOS AÑOS OCHENTA}

La recuperación de la democracia en 1983 concitó una serie de reacomodamientos en el escenario de la sociología local. En la Carrera de la UBA se produjo una profunda reorientación signada por la expulsión de los docentes que se habían hecho cargo de la institución en el período anterior y el regreso de un buen número de sociólogos que habían sido docentes en etapas previas. Para quienes volvían a la histórica pormenorizada de ese proceso y de esa etapa histórica, puede verse Blois (2009)
Carrera se trataba de recuperar un espacio que, en su mirada, había sido vaciado de "contenido sociológico". ${ }^{6}$ Conscientes de la situación de virtual aislamiento que había caracterizado a la institución durante la dictadura militar, las nuevas autoridades se propusieron conectar a los 
estudiantes con los problemas y necesidades de la sociedad en sus más diversos ámbitos. En función de ello, buscaron promover una concepción de la sociología que no se restringiera a las labores académicas. En su visión, se trataba de restablecer un vínculo seriamente afectado por la censura y persecución política.

Con eso en mente, una de las primeras iniciativas fue establecer como un requisito obligatorio para todos los estudiantes la realización de una práctica preprofesional o pasantía. Esa iniciativa, impulsada por una comisión convocada para diseñar un nuevo plan de estudios -la "Comisión Asesora Pedagógica"-, recuperaba algunas de las orientaciones que en el pasado habían promovido los defensores de la "sociología aplicada" y su encendido énfasis en la construcción de clientelas y empleadores, pero también las preocupaciones y experiencias de quienes se habían reunido en torno al CGS.

Según la flamante Comisión, las prácticas preprofesionales podrían contribuir a la familiarización de los alumnos con las “problemáticas que presenta la vida social de la Argentina actual” y facilitar su posterior inserción en el mercado de trabajo. Para ello instaba al establecimiento de convenios formales con dependencias estatales e instituciones de la sociedad civil. De acuerdo a su diagnóstico, la interacción con el "afuera” no sólo aseguraría la relevancia e incidencia social de las iniciativas de los sociólogos, sino que constituiría una fuente de preguntas y preocupaciones indispensables para su labor intelectual. La prédica de la Comisión era inseparable de una visión sobre el Estado y la sociedad civil que, si bien buscaba distinguirse de aquella que había informado la creación de la Carrera en los años cincuenta, tenía una fuerte afinidad con ella. ${ }^{7}$ En ambas, el sociólogo debía estar formado para satisfacer las demandas de la sociedad y del Estado, y su labor no debía asumirse como una práctica puramente académica, realizada como un fin en sí mismo. La disciplina, según se esperanzaba la Comisión, podría tener un rol central en los tiempos venideros. de los graduados en el Estado, la Comisión aclaraba: "no nos referimos a la figura del planificador prevaleciente en las décadas del 50 y el 60 , sino a la participación en procesos de elaboración de políticas estatales [...] que resguarde el pluralismo evitando la ilusión ideológica del gran planificador poseedor de una racionalidad superior" (COMISIÓN ASESORA PEDAGÓGICA, 1985, p. 4). 
Con todo, las prácticas preprofesionales no pudieron ser instrumentadas. Por un lado, la situación heredada no era la más propicia para semejante desafío: a la escasez de recursos económicos y el absoluto predominio de las dedicaciones simples, se sumaba el marginamiento propio de una institución que había sido fuertemente degradada en los años previos, funcionando como indicamos en un estado de virtual aislamiento, sin relaciones con instituciones de la sociedad civil o el Estado que pudieran facilitar el establecimiento de acuerdos de cooperación (BLOIS, 2009). En este caso, como en otros, el impacto de la dictadura había sido mucho más profundo de lo que inicialmente se pensó, limitando las posibilidades de una pronta recuperación.

Por el otro, en el clima de entusiasmo suscitado por la vuelta de la democracia y la recuperación de los espacios de militancia política, las miradas que en el pasado habían recusado la preocupación por la salida laboral y el estímulo de la sociología como una “profesión”, presentes en las disposiciones o formas de entender la disciplina de una buena parte de los docentes que volvían a la Carrera, encontraron un extendido eco entre un estudiantado mucho más interesado en conectar con la agenda de discusiones políticas e ideológicas del momento que en su futuro laboral. Para quienes se ilusionaban con las profundas transformaciones que la nueva etapa podría inaugurar, la cuestión laboral no era más que una distracción o tema menor. En sus cuestionamientos a las prácticas preprofesionales, los estudiantes y una parte de los profesores recuperaban la oposición que organizó buena parte de las discusiones del pasado entre una sociología más “politizada” y una sociología más "profesional". El nuevo plan, aprobado en 1988 y vigente desde entonces, dejó de lado la preocupación por las salidas laborales y la vinculación con el mundo del trabajo (BLOIS, 2009).

A partir de los años noventa, las inserciones profesionales de los sociólogos experimentaron profundas transformaciones (BLOIS, 2012; BELTRÁN, 2010; RUBINICH; BELTRÁN, 2010). A la ampliación de las instituciones académicas, se sumó un conjunto de instituciones no académicas que comenzaron a contratar un número creciente de graduados. Dependencias estatales, consultoras, grandes empresas privadas y ONGs ofrecieron nuevas oportunidades y propiciaron un notable crecimiento y diferenciación de las prácticas profesionales de los sociólogos. Mientras una parte (minoritaria) de los graduados encontraba su inserción principal en las instituciones académicas, el resto, por necesidad o elección, desembarcaba en alguno de estos espacios. $^{8}$

Ahora bien, frente a ese escenario de profundas transformaciones, la Carrera de la UBA se mantuvo relativamente ajena o cerrada. Ni la preocupación por la inserción profesional de sus estudiantes ni las experiencias variadas que buena parte de sus graduados fueron 
desarrollando, encontraron un ámbito propicio para su procesamiento institucional (BLOIS, 2012; BONALDI, 2009). Si, como mencionamos, inicialmente el contexto de activación política vinculado a la salida de la dictadura había dejado en un plano muy secundario la pregunta por el futuro laboral, luego, cuando el entusiasmo inicial fue dando paso a la desilusión por las promesas incumplidas de la democracia, la situación no varió.

Con el cambio de década y el despliegue de las iniciativas neoliberales, la reluctancia a introducir cambios en la currícula aparecía para buena parte de los docentes y alumnos como un acto de "resistencia" frente a las políticas emanadas desde los organismos financieros internacionales. Para quienes confluían en ese espacio, el planteo de la cuestión laboral podía ser visto como una "instrumentalización” de la formación en función de las necesidades del "mercado". En ese marco, sólo la esfera académica aparecía como el ámbito capaz de asegurar la autonomía que el ejercicio “crítico” de la disciplina requería. A veces en las clases se mencionaban las otras posibilidades de inserción, pero por lo general eran subestimadas o desvalorizadas frente al ideal del sociólogo como docente e investigador universitario (BONALDI, 2009). El claustro de los graduados, supuestamente destinado a conectar la formación con los problemas y desafíos del mundo del trabajo, estuvo muchas veces orientado a otras preocupaciones. Por su parte, las materias optativas, que se fueron multiplicando, tampoco sugerían en términos generales caminos distintos al académico, aun cuando muchos de sus docentes-en un carrera donde predominaban las dedicaciones simples- tenían como inserción principal una inserción fuera de la academia (BLOIS, 2009). Los cambios en el mundo del trabajo, pese a su alcance y magnitud, tuvieron en esas condiciones poco impacto en la manera en que las nuevas generaciones de sociólogos eran formados.

En esas condiciones, no debería sorprender que para muchos graduados la inserción en una esfera laboral no académica conllevara un ostensible distanciamiento de aquello que esperaban realizar una vez finalizados los estudios. Si bien es posible suponer que hay siempre una relativa distancia entre formación universitaria y ejercicio profesional, propia de la transición al mundo del trabajo, la magnitud que asumía en esos casos resultaba particularmente marcada. Lejos de percibir que en su práctica profesional aplicaban lo que habían aprendido en su paso por la universidad, estos sociólogos experimentaban una marcada "ruptura" o "quiebre" entre una instancia y otra. Esa crisis, vivida por lo general como algo personal o "psicológico", era el fruto de la distancia entre la idea de sociología incorporada durante la realización de sus estudios y aquello que efectivamente hacían en su trabajo cotidiano. ${ }^{9}$

Para un análisis de la forma en que el proceso de socialización universitaria de la Carrera de Sociología de la UBA impacta en las prácticas profesionales de sus graduados, puede verse Blois (2012). 


\section{REFLEXIONES FINALES}

A partir de la creación de la Carrera de Sociología de la UBA y a medida que el número de graduados fue en aumento, las disputas entre los sociólogos en torno a la definición de su disciplina se multiplicaron. Para algunos, la sociología debía constituirse como una “profesión” capaz, como el resto de las profesiones, de dar respuestas a las demandas de una clientela en base a un conjunto de saberes y técnicas específicos, orientada por una particular deontología. Para otros, que reaccionaban contra el ideal de la neutralidad valorativa propio de aquella posición, la asunción de la sociología como una "profesión” era una forma velada de poner a la disciplina al servicio de las elites políticas y sociales, únicas capaces de contratar los servicios de los sociólogos. Para estos últimos, la sociología debía estrechar sus vínculos con la práctica política y la crítica de las ideologías. Más aún, en las versiones más extremas el sociólogo debía asumirse como un militante político. Ahora bien, sea como fuesen que definiesen sus alcances, para unos tanto como para los otros, la sociología estaba destinada a cumplir un papel muy gravitante en el desarrollo y transformación de la sociedad. La discusión en torno a las inserciones laborales de los sociólogos aparecía como una cuestión subordinada a aquella definición más amplia.

El quiebre introducido desde mediados de los años setenta con la instauración del autoritarismo y la violencia estatal impuso un contexto ciertamente hostil para la sociología, visualizada por buena parte de las autoridades gubernamentales como una expresión más de la "subversión” que se habían propuesto eliminar. Ese marco, como vimos en el caso del CGS, alentó una mirada más estrecha sobre el papel de los sociólogos en la sociedad y su relación con el mundo del trabajo. Al tiempo que se tomaba distancia de los discursos más “politizados” del período anterior, se recuperaba el énfasis de quienes habían promovido la "sociología aplicada” pero con una orientación más "pragmática”: los sociólogos tenían que ajustar sus servicios a aquello que la sociedad demandara, dejando de lado las expectativas más ambiciosas que los habían orientado en el pasado.

La vuelta de la democracia alentó una profunda reorientación en el espacio de la sociología y un renovado entusiasmo sobre las posibilidades de la disciplina en el nuevo contexto. En la Carrera de Sociología de la UBA hubo quienes alentaron una fluida conexión con el mundo del trabajo, esperanzados con el aporte que la disciplina podría hacer en la nueva hora. La iniciativa de las prácticas preprofesionales no sólo buscaba facilitar la inserción laboral de los futuros sociólogos sino que, ante todo, buscaba regenerar una mayor vinculación de la disciplina con las necesidades y problemáticas de la sociedad. Sin embargo, las condiciones heredadas del período de la dictadura no serían fácilmente remontables: la Carrera era una institución fuertemente marginalizada 
en el espacio universitario y sin demasiados recursos institucionales y humanos para promover esas prácticas. Por otra parte, rápidamente se dio una recuperación de las posiciones más críticas de la sociología como “profesión” motorizada en buena medida por una parte de los docentes y los estudiantes, más identificados con la figura del intelectual público o el militante político que con la del técnico o experto. Desde entonces, el perfil de la Carrera -que mostró una notable estabilidad representada de modo emblemático en la vigencia de un plan de estudios que no se modifica desde 1988- tendió a privilegiar la inserción académica como destino deseable de los futuros sociólogos y a desconocer las otras opciones; opciones que, sin embargo, a partir de los años noventa se fueron multiplicando al calor de las profundas transformaciones que vivía la sociedad argentina.

En el pasado una de las razones por las que la vocación por promover la inserción de los sociólogos más allá de la vida académica había naufragado era la debilidad del mercado de trabajo. Ahora, en contraste, las oportunidades laborales estaban, pero faltaba la disposición institucional para incorporar esas experiencias. La idea de una sociología académica y una sociología desarrollada para un cliente o empleador como dos quehaceres entre los que no había demasiados cruces posibles tuvo una fuerte gravitación y orientó el proceso de socialización universitario de los sociólogos que se daba en la UBA. Sin dudas, esa idea, que reactualizaba en el nuevo contexto el desprecio hacia la "sociología aplicada" de otros momentos, contribuía a reforzar el perfil libresco de la enseñanza, al tiempo que desestimulaba una mayor conexión de la institución con las problemáticas más “concretas” e inmediatas de la sociedad con las que una buena parte de sus graduados trabajaban cotidianamente.

Por supuesto, las tensiones de la sociología a la hora de relacionarse con el mundo del trabajo no son una peculiaridad del caso argentino. Lejos de ello, la vocación crítica y cuestionadora de las relaciones de poder que anida en la sociología desde sus mismos orígenes ha sido movilizada una y otra vez contra la concepción que hace de la sociología una técnica capaz de "racionalizar" las acciones de aquellos que están dispuestos a contratar sus servicios (DUBET, 2012). La sociología, según una célebre fórmula, debe ser una "ciencia que incomoda" y ello no siempre facilita el trabajo para un cliente o empleador, poco preocupados por develar los mecanismos de dominación y las asimetrías de poder. Ahora bien, la configuración de una Carrera que, pese a los enormes cambios que se daban en el mercado de trabajo, se mantuvo virtualmente cerrada a esas experiencias, o que transmitía una idea de la sociología ceñida de modo casi exclusivo a la vida académica aun cuando la mayoría de sus docentes se ganaba la vida trabajando en empresas, dependencias estatales y otras organizaciones, constituye una realidad particular, cuya conformación sociohistórica nos propusimos aquí recorrer. 


\section{REFERENCIAS}

BEIGEL, Fernanda. La institucionalización de las ciencias sociales en América Latina: entre la autonomía y la dependencia académica. In: BEIGEL, Fernanda (Dir.). Autonomía y dependencia académica. Universidad e investigación científica en un circuito periférico: Chile y Argentina (1950-1980). Buenos Aires: Biblos, 2010. p. 47-64.

BELTRÁN, Gastón. Las ciencias sociales y el surgimiento de un mercado del saber experto. Las bifurcaciones de la sociología argentina en el final del siglo XX. In: RUBINICH, L.; BELTRÁN, G. (Ed.). ¿Qué hacen los sociólogos? Buenos Aires: Aurelia, 2010. p. 46-78.

BIALAKOWSKY, Alberto et al. Espectro ocupacional del licenciado en sociología en el medio profesional argentino. Buenos Aires: Colegio de Graduados en Sociología, 1982.

BLANCO, Alejandro. Razón y modernidad. Gino Germani y la sociología en la Argentina, Buenos Aires: Siglo XXI, 2006.

BLANCO, Alejandro; JACKSON, Luiz. “Jefes de escuela” en la sociología latinoamericana: Gino Germani, Florestan Fernandes y Pablo González Casanova. Sociológica, México D.F., v. 32, n. 90, p. 9-46, enero/abr. 2017.

BLOIS, Pedro. Sociología y democracia: la refundación de la carrera de sociología de la Universidad de Buenos Aires (1984-1990). Sociohistórica, La Plata, n. 26, p. 111-150, 2009.

BLOIS, Pedro. Obligados a elegir entre el "sacerdocio y la prostitución". Socialización universitaria y prácticas profesionales de los sociólogos de la UBA. Tese (Doutorado em Ciências Sociais) Universidad de Buenos Aires, Buenos Aires, 2012.

BLOIS, Pedro. La institucionalización de la sociología en Brasil y Argentina. Estudios Sociológicos, México D. F., v. 33, n. 99, p. 633-658, 2015.

BLOIS, Pedro. Las disputas por la definición de la sociología en la Argentina. Horizontes Sociológicos, Buenos Aires, v. 4, n. 8, p. 10-33, 2016.

BONALDI, Pablo. Aprendiendo sociología. Buenos Aires: La Gomera, 2009.

BONELLI, Maria. Identidade profissional e mercado de trabalho dos cientistas sociais. Tese (Doutorado em Sociologia) - Universidade Estadual de Campinas, Campinas, 1993.

BRAGA, Eugênio. Novos elementos para uma sociologia dos cientistas sociais. A situação ocupacional dos egressos. Revista Brasileira de Ciências Sociais, São Paulo, v. 26, n. 76, p. 104-122, 2011.

BRUNNER, José; BARRIOS, Alicia. Inquisición, mercado y filantropía. Santiago de Chile: FLACSO, 1987.

BURAWOY, Michael. Por una sociología pública, Política y sociedad, Madrid, v. 42, n. 1, p. 197-225. 2005.

CALHOUN, Craig; WIEVIORKA, Michel. Manifiesto por las ciencias sociales, Revista Mexicana de Ciencias Políticas y Sociales, México D. F., v. 58, n. 217, p. 29-59. 2013.

CARRI, Roberto. El formalismo en las ciencias sociales (2da. parte). Antropología del tercer mundo, Buenos Aires, n. 2, p. 55-64. 1969.

CARRI, Roberto. Obras completas, tomo 2. Buenos Aires: Biblioteca Nacional, 2015.

COMISIÓN ASESORA PEDAGÓGICA. Proyecto de Reforma Curricular de la Carrera de Sociología de la Universidad de Buenos Aires. 1985. Minografado.

DUBAR, Claude Préface. In: PIRIOU, O. La face cachée de la sociologie. Paris: Belin, 2006. p. 7-12.

DAGFAL, Alejandro. Entre París y Buenos Aires. Psicología, psicoanálisis y psiquiatría (1942-1966). Buenos Aires: Paidós, 2009.

DE ÍMAZ, José Luis et al. Del sociólogo y su compromiso. Buenos Aires: Libera, 1966.

DE VENANZI, Augusto. La sociología de las profesiones y la sociología como profesión. Caracas:

Universidad Central de Venezuela, 2003. 
DI TELLA, Torcuato. La sociología y la praxis social. Revista Latinoamericana de Sociología, Buenos Aires, v. 3, n. 1, p. 84-90, 1967.

DI TELLA, Torcuato. La sociología argentina en una perspectiva de veinte años. Desarrollo Económico, Buenos Aires, v. 20, n. 79, p. 299-327, 1980.

DUBET, François. ¿Para qué sirve un sociólogo? Buenos Aires: Siglo XXI, 2012.

FACULTAD DE FILOSOFÍA Y LETRAS UBA. Guía de informaciones. Buenos Aires, 1962.

GERMANI, Ana. Gino Germani: del antifascismo a la sociología. Buenos Aires: Taurus, 2004.

GERMANI, Gino. La sociología científica. Apuntes para su fundamentación. 2. ed. México: UNAM, 1962. 1. ed. 1956.

GERMANI, Gino. O profesor e a cátedra. América Latina, Rio de Janeiro, v. 13, n. 1, p. 83-101, jan./mar. 1970.

GERMANI, Gino; GRACIARENA, Jorge. Estudio preparado para el Seminario Latinoamericano sobre Metodología de la Enseñanza y la Investigación, organizado por UNESCO, FLACSO y Centro Latinoamericano de Investigaciones en Ciencias Sociales. Buenos Aires: UBA, 1958.

GIORGI, Guido. Una aproximación histórica a la sociología como profesión (1940-1955). In: JORNADAS DE SOCIOLOGÍA UNLP, 5., 2010, La Plata. Anais... La Plata: UNLP, 2010.

GONZÁLEZ BOLLO, Hernán. El nacimiento de la sociología empírica en la Argentina: El Instituto de Sociología, Facultad de Filosofía y Letras (UNA), 1940-54. Buenos Aires: Dunken, 1999.

GRISENDI, Ezequiel. Del barrio a la región: Adolfo Critto y la investigación social aplicada en Córdoba (1963-1975). In: JORNADAS DE SOCIOLOGÍA UNLP, 8., 2010, La Plata. Anais... La Plata: UNPL, 2014.

HEREDIA, Mariana. Los centros privados de expertise en economía: génesis, dinámica y continuidad de un nuevo actor político en la Argentina. In: MORRESI, S.; VOMMARO, G. (Comp.). Saber lo que se hace. Expertos y política en Argentina. Buenos Aires: Prometeo-UNGS, 2011. p. 132-156.

LAHIRE, Bernard. ¿Para qué sirve la sociología? Buenos Aires: Siglo XXI, 2006.

LEGRAND, Monique; VRANCKEN, Didier. L'espertise du sociologue. Paris: L'Harmattan, 2004.

LIEDKE FILHO, Enno. Sociology and Society in Brazil and Argentina 1954-1985. Tese (Doutorado) Brown University, Providence, 1991.

MACHUCA, Adriana. La identidad profesional de los sociólogos. 2008. Dissertação (Mestrado em Sociologia) - FLACSO, México D.F., 2008.

MILANESIO, Natalia. Cuando los trabajadores salieron de compras. Buenos Aires: Siglo XXI, 2014.

MORA Y ARAUJO, Manuel. La sociedad y la praxis sociológica. Desarrollo Económico, Buenos Aires, v. 11, n. 41, p. 125-143, 1971.

NOÉ, Alberto. Utopia y desencanto. Creación e institucionalización de la carrera de sociología de la Universidad de Buenos Aires: 1955-1966. Buenos Aires: Miño y Dávila, 2005.

PEREL, Pablo et al. Universidad y dictadura. Buenos Aires: CCC, 2006.

PEREYRA, Diego. International Networks and the Institutionalization of Sociology in Argentina (19401963). Tese (Doutorado em Sociologia) - University of Sussex, Brighton, 2005.

PEREYRA, Diego et al. Atención, sociólogos trabajando. Desafíos de la inserción profesional de los primeros sociólogos y sociólogas en Argentina (1961-1985). Política \& Sociedade, Florianópolis, v. 14, n. 31, p. 227-255, 2016.

PERLATTO, Fernando; MAIA, João Marcelo. Qual sociología pública? Uma visão a partir da periferia. Lua Nova, São Paulo, n. 87, p. 83-112, 2012.

PICÓ, Joseph. Los años dorados de la sociología. Madrid: Alianza, 2003.

PIRIOU, Odile. La face cachée de la sociologie. Paris: Belin, 2006. 
RAUS, Diego. La sociología en el "Proceso". Sociología en Debate, Buenos Aires. n. 1, p. 35-48. 2007.

RIBEIRO, Adelia. A sociologia quando "sai" da universidade: ilustrações para um debate. Cadernos Ceru, s. 2, n. 10, p. 173-186, 1999.

RODRÍGUEZ BUSTAMANTE, Norberto. Sociology and reality in Latin America, the case of Argentina. International Social Science Journal, v. 31, p. 86-97, 1979.

RUBINICH, Lucas. Los sociólogos intelectuales: cuatro notas sobre la sociología en los ‘60. Apuntes de Investigación del CECyP, Buenos Aires, n. 4, 1999.

RUBINICH, Lucas. Cómo relatar aquello que hacen los sociólogos. In: RUBINICH, L.; BELTRÁN, G. (Ed.). ¿Qué hacen los sociólogos? Buenos Aires: Aurelia, 2010.

SIDICARO, Ricardo. Reflexiones sobre la accidentada trayectoria de la sociología en la Argentina. Cuadernos Hispanoamericanos, Madrid, n. 517-519, p. 65-76, 1993.

SIGAL, Silvia. Intelectuales y poder en Argentina. Buenos Aires: Puntosur, 1991.

TURNER, Jonathan. Contra a sociologia pública: será ela a melhor forma de tornar a sociologia relevante?. Caderno CRH, Salvador, v. 22, n. 56, p. 255-269, 2009.

VERÓN, Eliseo. Imperialismo, lucha de clases y conocimiento: 25 años de sociología en Argentina. Buenos Aires: Tiempo Contemporáneo, 1974.

\section{JUAN PEDRO BLOIS}

Universidad Nacional de General Sarmiento -UNGS-, Los Polvorines, Buenos Aires, Argentina; Consejo Nacional de Investigaciones Científicas y Técnicas -Conicet-, Buenos Aires, Argentina

pedro.blos@gmail.com 